Applied Remote Sensing

Errata: Fast image-formation algorithm for ultrahigh-resolution airborne squint spotlight synthetic aperture radar based on adaptive sliding receive-window technique

Wei Yang

Hong-cheng Zeng

Jie Chen

Peng-bo Wang 


\title{
Errata: Fast image-formation algorithm for ultrahigh-resolution airborne squint spotlight synthetic aperture radar based on adaptive sliding receive-window technique
}

\author{
Wei Yang, Hong-cheng Zeng, Jie Chen, and Peng-bo Wang \\ Beihang University, School of Electronics and Information Engineering, Xueyuan Road 37, \\ Beijing 100191, China
}

[DOI: $10.1117 / 1 . J R S .8 .089998]$

This article [J. Appl. Remote Sens. 8(1), 083620 (June 04, 2014)] contained errors in the authors' names. The corrected names appear above. Online versions of the article were corrected on 6 June 2014. 